\title{
Alterações eletrocardiográficas na intoxicação aguda por antidepressivo tricíclico e suas implicações clínicas
}

\section{Electrocardiographic alterations in acute tricyclic antidepressant intoxication and its clinical implications}

\author{
Carlos H. Miranda' ${ }^{1}$ Paula M. Luciano², Andrés R. P. Riera ${ }^{3}$, Antônio Pazin Filho ${ }^{4}$
}

\begin{abstract}
RESUMO
Introdução: A intoxicação aguda por antidepressivos tricíclicos é comum nas salas de emergência. Anormalidades no eletrocardiograma de 12 derivações (ECG) são associadas a este tipo de intoxicação, com implicações diagnósticas e prognósticas. O ECG pode ter valor incremental principalmente em situações de alteração de consciência, convulsões ou distúrbios do ritmo cardíaco associado.

Objetivo: Revisar as principais alterações eletrocardiográficas descritas na intoxicação por antidepressivos tricíclicos e estabelecer suas implicações prognósticas no manejo desta condição clínica.

Metodologia: Pesquisa bibliográfica no MEDLINE (PubMEd) limitada aos artigos publicados entre 1980 e 2010 em língua inglesa, utilizando-se unitermos (tricyclic antidepressant overdose OR tricyclic antidepressant intoxication OR tricyclic antidepressant poisoning OR tricyclic antidepressant ingestion AND electrocardiography). Dos 133 artigos identificados, foram selecionados 44 para a revisão.

Resultado: Foram identificados como alterações prevalentes com poder diagnóstico em ordem decrescente os seguintes achados: desvio dos $40 \mathrm{~ms}$ terminais do QRS para a direita do plano frontal $(61,0-$ $83,0 \%)$, taquicardia sinusal $(40,7-57,7 \%)$, prolongamento do QTc (8,2-53,0\%), relação R/S em aVR>0,7 $(28,8-32,9 \%)$, prolongamento do QRS $(8,2-21,3 \%)$ e padrão Brugada-like $(2,3-15,3 \%)$. Destes achados, somente o prolongamento do QRS foi prognóstico para morte, convulsão e arritmia e o prolongamento do QTc e a relação R/S em aVR $>0,7$ para arritmia ventricular. Para o padrão Brugada-like, as evidências são escassas, mas sugestivas de maior chance de convulsão e hipotensão.

Conclusão: O ECG é um instrumento de fácil realização na sala de urgência, fornecendo informações diagnósticas e prognosticas para a intoxicação por tricíclicos, devendo ser realizado em todo paciente com suspeita desta intoxicação.
\end{abstract}

Palavras-chaves: Antidepressivos Tricíclicos. Overdose. Intoxicação. Eletrocardiografia.

\section{Introdução}

Os antidepressivos tricíclicos (ADT) começaram a ser utilizados na prática clínica na década de 50 .
Inicialmente, esta medicação era utilizada no tratamento dos transtornos depressivos e atualmente, houve incremento da sua utilização em outras entidades clínicas tais como as dores neuropáticas, distúrbios fun-
${ }^{1}$ Médico Assistente da Unidade Coronariana da Unidade de Emergência do Hospital das Clínicas da Faculdade de Medicina de Ribeirão Preto da Universidade de São Paulo

${ }^{2}$ Docente da Faculdade de Medicina da Universidade Federal de São Carlos - São Carlos - São Paulo.

${ }^{3}$ Docente da Faculdade de Medicina ABC - Santo André/ SP

${ }^{4}$ Docente da Divisão de Emergências Clínicas do Departamento de Clínica Médica da Faculdade de Medicina de Ribeirão Preto da Universidade de São Paulo - Ribeirão Preto/ SP
Correspondência:

Prof. Dr. Antônio Pazin Filho Unidade de Emergência do Hospital das Clínicas da Faculdade de Medicina de Ribeirão Preto - USP

Rua Bernardino de Campos, 1000 14010-030 - Ribeirão Preto - SP email: apazin@fmrp.usp.br

Artigo recebido em 18/02/2010 Aprovado em 06/07/2010 
cionais gastrointestinais dentre outros. Desta maneira a intoxicação exógena por antidepressivos tricíclicos continua frequente nas salas de urgência. ${ }^{1}$

Os ADT mais utilizados são: amitriptilina, imipramina, clomipramina e nortriptilina. A intoxicação por esta classe de medicação é uma situação clínica potencialmente grave e marcada por manifestações neurológicas e cardiovasculares, principalmente com risco de convulsões e arritmias cardíacas. ${ }^{2}$ A maior parte dos pacientes apresenta somente sedação e efeitos anticolinérgicos e se recuperam com cuidados de suporte, porém alguns pacientes podem apresentar uma deterioração rápida do seu estado clínico com risco de morte. ${ }^{3,4,5}$ Vários testes diagnósticos, entre eles, o eletrocardiograma convencional de 12 derivações (ECG), tentam selecionar estes pacientes com maior risco de complicações, que necessitam de uma monitorização e tratamento mais intensivo. ${ }^{6,7}$

O ECG desempenha um papel fundamental na abordagem destes pacientes, pois ajuda na suspeita diagnóstica de intoxicação por tricíclicos, principalmente nos estados comatosos, podendo ser útil na estratificação de risco de complicações como convulsões e arritmias cardíacas e também muitas vezes, orientar a intervenção terapêutica com bicarbonato de sódio. ${ }^{8-11}$

O objetivo deste artigo foi revisar as principais alterações eletrocardiográficas observadas na intoxicação aguda pelos ADT e avaliar seu valor diagnóstico e prognóstico.

\section{Métodos}

Foi realizado pesquisa bibliográfica no MEDLINE (PubMEd) limitada aos artigos publicados entre 1980 e 2010 em língua inglesa. Utilizaram-se como estratégia de pesquisa, os seguintes termos: tricyclic antidepressant overdose OR tricyclic antidepressant intoxication $\mathrm{OR}$ tricyclic antidepressant poisoning OR tricyclic antidepressant ingestion AND electrocardiography. Foram encontrados 133 artigos científicos. Os títulos e resumos foram analisados para identificação daqueles que abordaram especificamente alterações eletrocardiográficas na intoxicação aguda por antidepressivos tricíclicos. Após esta análise foram selecionados 44 artigos que foram avaliados integralmente. Para maior esclarecimento no assunto, as citações de outros trabalhos relevantes sobre o assunto foram consideradas a partir das referências iniciais.

As figuras utilizadas neste trabalho foram reproduzidas conforme autorização obtida por termo de consentimento do projeto CEP/HCFMRP-USP 11738/ 2004.

\section{Resultados}

As principais alterações eletrocardiográficas encontradas na intoxicação aguda por ADT são: taquicardia sinusal, prolongamento do intervalo PR, prolongamento do intervalo QT, prolongamento do QRS, desvio dos $40 \mathrm{~ms}$ terminais do QRS para a direita no plano frontal, aumento da amplitude da onda $\mathrm{R}$ em aVR, padrão eletrocardiográfico Brugada-like, bloqueios atrioventriculares, arritmias supraventriculares, arritmias ventriculares como extrassístoles ventriculares, taquicardia ventricular, fibrilação ventricular e até mesmo atividade elétrica sem pulso. ${ }^{12,13,14,15,16}$ (Tabela 1).

A ação desta classe de drogas (ADT) a nível celular do miócito, causando um bloqueio dos canais rápidos de sódio (efeito quinidina-like) é o mecanismo responsável pela maior parte das alterações eletrocardiográficas encontradas no episódio de intoxicação aguda. O bloqueio dos canais de potássio do tipo retificadores tardios $\left(\mathrm{I}_{\mathrm{kr}}\right)$ da membrana celular também pode ter um papel secundário no desencadeamento destas alterações eletrocardiográficas. ${ }^{12}$

\section{Taquicardia sinusal}

A frequência cardíaca acima de 100 bpm é uma alteração muito frequente. Demonstrou-se em um grupo de pacientes com suspeita de intoxicação, que a frequência cardíaca foi maior naqueles onde a dosagem toxicológica para ADT foi positiva $(117 \pm 23 \mathrm{bpm})$ em relação aqueles com dosagem negativa $(103 \pm 15$; $\mathrm{p}<0,05)$. Os principais mecanismos responsáveis por essa taquicardia são o efeito anticolinérgico e a inibição da recaptação da noradrenalina pelos ADT. Apesar da alta prevalência desta alteração, a mesma apresenta baixa especificidade para identificação destes pacientes, visto que inúmeras outras intoxicações ou afecções clínicas podem cursar com taquicardia. Nenhum estudo avaliou impacto prognóstico da taquicardia sinusal. ${ }^{11,13}$

\section{Desvio dos 40ms terminais (T40ms) do QRS para a direita no plano frontal}

Esta alteração eletrocardiográfica foi inicialmente descrita em 1986, observando-se que pacientes com intoxicação por ADT apresentam desvio para direita dos $40 \mathrm{~ms}$ terminais do QRS no plano frontal $\left(195 \pm 51^{\circ}\right)$ em relação aos indivíduos controles $\left(54 \pm 64^{\circ} ; \mathrm{p}<0,001\right)$. 


\section{Tabela 1}

Prevalência, sensibilidade, especificidade e likelihood ratio positivo para predizer o risco de complicações (morte, convulsões e arritmia ventricular) das principais alterações eletrocardiográficas na intoxicação por ADT

\begin{tabular}{|c|c|c|c|c|c|}
\hline Alterações ECG & $\begin{array}{l}\text { Prevalência } \\
(\%)\end{array}$ & $\begin{array}{c}\text { Complicação } \\
\text { predita }\end{array}$ & $\begin{array}{l}\text { Sensibilidade } \\
\quad(95 \% \mathrm{Cl})\end{array}$ & $\begin{array}{l}\text { Especificidade } \\
\quad(95 \% \mathrm{Cl})\end{array}$ & $\begin{array}{l}\text { Likelihood } \\
\text { ratio positivo } \\
\quad(95 \% \mathrm{Cl})\end{array}$ \\
\hline Taquicardia sinusal & $40.7-57.7$ & NA & NA & NA & NA \\
\hline Prolongamento do $\mathrm{QRS}^{\dagger}$ & $8.2-21.3$ & Morte & $0.81(0.54-0.94)$ & $0.62(0.55-0.68)$ & $2.13(1.59-2.86)$ \\
\hline \multirow[t]{2}{*}{$(>100 \mathrm{~ms})$} & & Convulsões & $0.69(0.57-0.78)$ & $0.69(0.58-0.78)$ & $3.18(2.60-3.90)$ \\
\hline & & Arritmia Ventr. & $0.79(0.58-0.91)$ & $0.46(0.35-0.59)$ & $1.46(1.21-1.77)$ \\
\hline QTc prolongamento $^{\dagger}$ & $8.2-53.0$ & Morte & $0.50(0.00-0.91)$ & $0.68(0.22-0.94)$ & $1.56(0.36-6.75)$ \\
\hline \multirow[t]{2}{*}{$(>430 \mathrm{~ms})$} & & Convulsões & NA & NA & NA \\
\hline & & Arritmia Ventr. & $0.78(0.41-0.95)$ & $0.56(0.38-0.73)$ & $1.77(1.26-2.50)$ \\
\hline \multirow{3}{*}{$\begin{array}{l}\text { Desvio T } 40 \text { ms do QRS } \\
\text { para direita no plano frontal }^{\dagger}\end{array}$} & $61.0-83.0$ & Morte & $0.33(0.06-0.79)$ & $0.71(0.64-0.77)$ & $1.14(0.23-5.72)$ \\
\hline & & Convulsões & $0.50(0.19-0.81)$ & $0.72(0.64-0.77)$ & $1.74(0.76-4.00)$ \\
\hline & & Arritmia Ventr. & $0.33(0.06-0.79)$ & $0.71(0.64-0.77)$ & $1.14(0.23-5.72)$ \\
\hline Relação R/S em aVR $>0.7$ ‡ & $28.8-32.9$ & Arritmia Ventr. & $0.47(0.25-0.70)$ & $0.97(0.92-0.99)$ & $15.7(4.60-54.78)$ \\
\hline \multirow[t]{4}{*}{ Padrão Brugada-like } & $2.3-15.3$ & Morte & $*$ & $*$ & $*$ \\
\hline & & Convulsão & $0.08(0.01-0.23)$ & $0.98(0.96-0.99)$ & 5.2 \\
\hline & & Arritmia Ventr. & $*$ & $*$ & $*$ \\
\hline & & Hipotensão & $0.08(0.02-0.18)$ & $0.98(0.97-0.99)$ & 7.12 \\
\hline
\end{tabular}

Likelihood ratio positivo - medida semi-quantitativa do desempenho de um teste diagnóstico, indica se a alteração esta presente, o quando ela modifica a probabilidade pré-teste; NA - parâmetro não avaliado; Arritmia Ventr. - Arritmia ventricular; (*) não preditor para estas complicações; ${ }^{\dagger}$ dados obtidos de meta-análise ${ }^{40}$; ${ }^{*}$ dados obtidos de estudos isolados 19,37

Este desvio pode ser facilmente avaliado no eletrocardiograma através de ondas $\mathrm{S}$ profundas em DI e onda $\mathrm{R}$ proeminentes em aVR na ausência de bloqueio de ramo direito. A alteração acima descrita parece ser altamente prevalente nestes pacientes $(83 \%) .{ }^{11}$ (Figura 1)

A presença de um desvio do T40ms do QRS para a direita no plano frontal para identificação dos pacientes intoxicados por ADT apresentou uma sensibilidade de $83 \%$ e especificidade de $63 \%$. Utilizando-se de curva ROC encontrou-se que o desvio do T40ms do QRS para direita no plano frontal foi um melhor indicador que o prolongamento do QRS para a identificação destes pacientes $(\mathrm{p}<0.05)$. Apesar disto, não foi encontrada correlação entre o grau de desvio do T40ms do QRS para a direita e a concentração plasmática de ADT (r=0.04). ${ }^{17,18}$

$\mathrm{O}$ emergencista deve sempre estar atento para alteração deste tipo, pois na presença desta anormalidade no ECG deve-se suspeitar de intoxicação aguda por ADT. ${ }^{15} \mathrm{O}$ ECG pode ser a única ferramenta disponível para a suspeita diagnóstica destes casos em muitas unidades de saúde onde não são realizadas dosagens toxicológicas de rotina. 


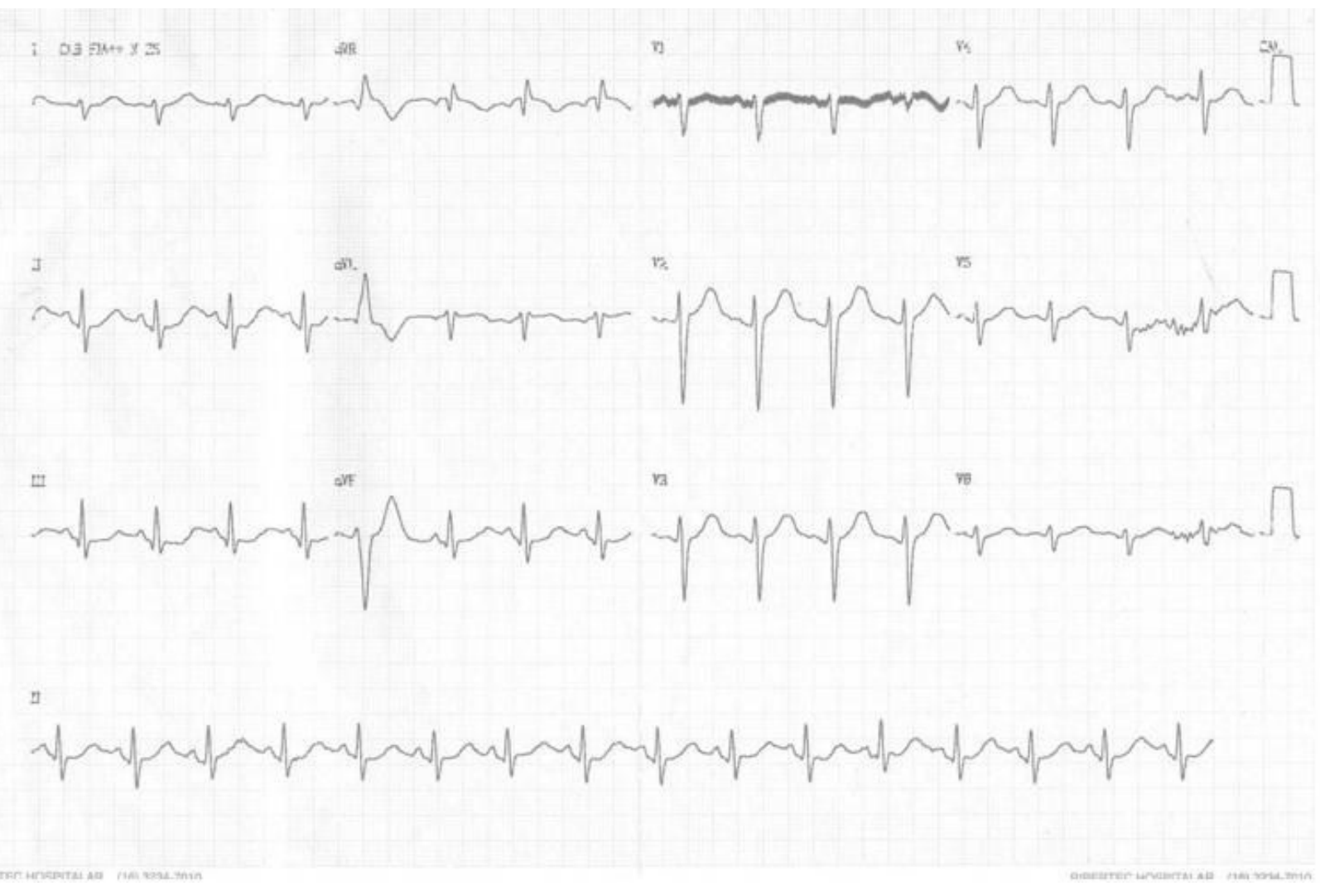

Figura 1: ECG de jovem intoxicado por ADT mostrando: ritmo sinusal, uma extrassístole ventricular isolada e desvio dos T40ms no eixo frontal para a direita caracterizado pela onda $S$ profunda em DI e a onda R ampla em aVR na ausência de bloqueio de ramo direito. A intoxicação por ADT foi confirmada por dosagem toxicológica. Reproduzido conforme autorização obtida por termo de consentimento do projeto CEP/HCFMRP-USP 11738/2004.

\section{Aumento da amplitude da onda R em aVR}

Ao avaliar pacientes no período das primeiras 24 horas após ingestão de ADT observou-se que aqueles que desenvolveram convulsões ou arritmias apresentam ondas $\mathrm{R}$ em aVR maiores que aqueles que não apresentaram estas complicações ( $4.4 \mathrm{~mm}$ versus $1.8 \mathrm{~mm}$; $\mathrm{p}<0.001$ ), assim como a relação entre a amplitude das ondas $\mathrm{R}$ e $\mathrm{S}$ em aVR (R/S) também foi maior neste primeiro grupo de pacientes (1.4 versus $0.5 ; \mathrm{p}<0.001) .{ }^{19}$

Utilizando-se de análise de regressão múltipla a presença de onda $\mathrm{R}$ em aVR maior que $3 \mathrm{~mm}$ foi à única variável capaz de predizer significativamente o risco de convulsões e arritmias (odds ratio: $6.9 ; 95 \% \mathrm{Cl}$ 1.2 a $40 ; p=0.03) .{ }^{19,23}$ (Figura 2)

\section{Prolongamento do QRS}

O prolongamento do complexo QRS com duração superior a $100 \mathrm{~ms}$ é frequente na intoxicação por ADT. Estudando 49 pacientes com intoxicação por
ADT não foi observado complicação neurológica ou arrítmica no grupo com duração do QRS inferior a 100 ms e incidências de $34 \%$ de crises convulsivas e $14 \%$ de arritmias naqueles com duração do QRS superior a $100 \mathrm{~ms}$. Convulsões ocorreram em pacientes com duração do QRS superior a $100 \mathrm{~ms}(\mathrm{p}<0.05)$, mas arritmias somente ocorreram naqueles com duração do QRS superior a $160 \mathrm{~ms}(\mathrm{p}<0.0005) .{ }^{20,21,22}$

Comparando-se a acurácia da duração do complexo QRS com a amplitude da onda R em aVR para avaliação do risco de convulsões e arritmias utilizando-se da curva ROC não foram observadas diferenças significativas $(\mathrm{p}=0.37)$ entre estas duas variáveis eletrocardiográficas. ${ }^{19}$ (Figura 2)

\section{Prolongamento do intervalo QT}

O prolongamento do intervalo QT é uma alteração eletrocardiográfica frequente nestes pacientes. ${ }^{10}$ Geralmente como eles apresentam frequências cardíacas elevadas na admissão, é necessária a correção do intervalo QT para a frequência cardíaca (QTc) uti- 


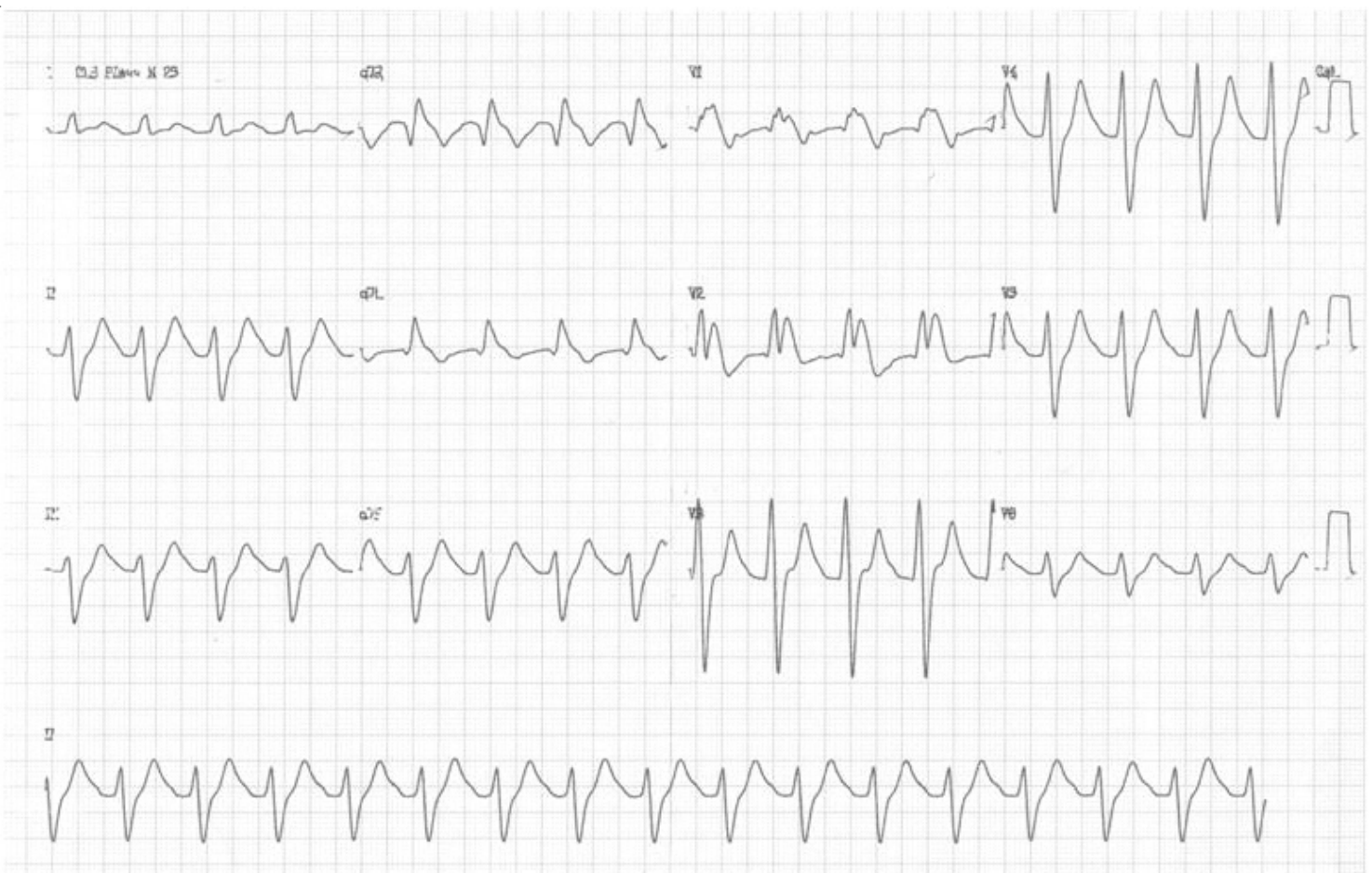

Figura 2: ECG de paciente intoxicado por ADT mostrando: taquicardia sinusal (100 bpm), importante alargamento do complexo QRS (220 $\mathrm{ms}$ ), proeminente onda $\mathrm{R}$ em aVR, padrão Brugada- like (bloqueio de ramo direito associado à supradesnivelamento do segmento ST em V1-V3). Este paciente apresentou crises convulsivas durante evolução. Reproduzido conforme autorização obtida por termo de consentimento do projeto CEP/HCFMRP-USP 11738/2004.

lizando-se de fórmulas como a de Bazett (QT c $=$ QT medido dividido pela raiz quadrada do intervalo $\mathrm{R}-\mathrm{R}$ precedente, em segundos). ${ }^{24}$

Pacientes com intoxicação por ADT, confirmados através de dosagem sanguínea, apresentam maiores valores do intervalo QT $(449 \pm 38 \mathrm{~ms})$ do que pacientes suspeitos de intoxicação na qual a dosagem de ADT foi negativa na corrente sanguínea $(418 \pm 36$ $\mathrm{ms} ; \mathrm{p}<0.05){ }^{11}$

O prolongamento do intervalo QT ocorre provavelmente devido a inibição dos canais de potássio pelos ADT. Poucos estudos avaliaram o impacto prognóstico do intervalo QT nesta situação, onde o aumento do intervalo QTc acima de $430 \mathrm{~ms}$ parece aumentar o risco de arritmias ventriculares (sensibilidade: $0.7895 \% \mathrm{Cl} 0.41$ a 0.95 e especificidade $0.5695 \%$ $\mathrm{Cl} 0.38$ a 0.73). ${ }^{40}$ Apesar de ser relativamente frequente o prolongamento do intervalo QT neste tipo de intoxicação, a ocorrência de taquicardia ventricular polimórfica associada (torsades de pointes) não é tão usual como esperada. Uma possível explicação para este fato seria que a bradicardia é um importante ga- tilho para o desencadeamento desta arritmia nos pacientes com intervalo QT aumentado, mas nos pacientes com intoxicação aguda por ADT geralmente observa-se importante taquicardia secundária ao efeito anticolinérgico do mesmo. ${ }^{25,26,27,28,29}$

\section{Padrão eletrocardiográfico Brugada-like}

A doença de Brugada é uma síndrome determinada geneticamente caracterizada pela morte súbita por arritmias ventriculares em pacientes com coração estruturalmente normal que apresentam um padrão eletrocardiográfico característico de bloqueio de ramo direito associado à supradesnivelamento do segmento ST nas derivações precordiais direitas (V1-V3). Esta doença esta associada à mutação no gene SCN5A que codifica a subunidade alfa dos canais rápido de sódio da membrana celular. ${ }^{30}$

Os pacientes com intoxicação por ADT podem desenvolver um padrão eletrocardiográfico semelhante ao descrito nesta síndrome genética, com o distúrbio de condução pelo ramo direito associado ao supradesnívelamento do segmento ST nas derivações 
precordiais direitas, isso provavelmente ocasionado pelo intenso bloqueio dos canais rápido de sódio causado por esta droga. ${ }^{31}$ (Figura 2)

O padrão Brugada-like parece ser pouco frequente durante a intoxicação por ADT $(2,3 \%)$. A mortalidade parece não ser diferente entre o grupo que desenvolveu esta alteração no eletrocardiograma (6.7\%) em relação ao grupo que não desenvolveu $(2.4 \%) \mathrm{p}=0.39$. A presença deste padrão eletrocardiográfico aumenta o risco de complicações como convulsões (RR $4.895 \% \mathrm{Cl} 1.8$ a 12.9) e hipotensão (RR $3.995 \% \mathrm{Cl} 2.1$ a 7.4), mas contraditoriamente ao esperado, não houve uma associação com o desenvolvimento de arritmias ventriculares. 32,33,34,35,36,37

Pacientes com o padrão Brugada-like apresentaram níveis séricos de ADT mais elevados em comparação com os indivíduos sem esta alteração $(\mathrm{p}=0.004)$ e geralmente ele desaparece quando a concentração plasmática de ADT cai abaixo de $1 \mu$ moles/L. ${ }^{32}$

\section{Bradicardia e bloqueios atrioventriculares}

Bradicardia e bloqueios atrioventriculares são raramente observados na intoxicação por ADT, este fato ocorre principalmente devido à ação anticolinérgica desta droga, sendo muito mais frequente a presença de taquicardia nesta situação. Entretanto os ADT, bloqueando os canais de sódio, podem afetar o funcionamento das células marca-passo encontradas principalmente no nódulo sinusal e nódulo atrioventricular causando bradicardia e bloqueios atrioventriculares. Estas alterações do ritmo cardíaco somente são observadas em graves intoxicações nas quais o bloqueio dos canais de sódio é tão profundo que superam a sua ação anticolinérgica e deste modo, a taquicardia não ocorre. A presença de bradiarritmias é um marcador de evolução desfavorável e risco iminente de parada cardiorrespiratória. ${ }^{12,13}$

\section{Arritmias ventriculares}

As drogas bloqueadoras dos canais de sódio podem causar alterações das propriedades eletrofisiológicas do tecido cardíaco, levando a um retardo na condução intraventricular do estímulo elétrico, propiciando a ocorrência de bloqueio unidirecional, que é uma parte fundamental para o estabelecimento dos circuitos de reentrada. Este mecanismo eletrofisiológico pode gerar as mais diversas arritmias cardíacas desde as extrassístoles ventriculares frequentes, até as taquicardias ventriculares sustentadas que podem posteriormente degenerar em fibrilação ventricular.
Estas arritmias ventriculares graves podem ocorrer em até $14 \%$ dos pacientes intoxicados e caracteriza uma das principais complicações associadas a este tipo de intoxicação, pois quando não identificadas e tratadas prontamente, podem levar a uma parada cardiorrespiratória. ${ }^{38,39}$

A ocorrência de arritmias supraventriculares também é relatada durante intoxicação por ADT, muitas destas arritmias supraventriculares são conduzidas com aberrância nesta situação, dificultando o diagnóstico diferencial com arritmias ventriculares. ${ }^{10}$

\section{Discussão}

O ECG é um exame complementar de baixo custo, de fácil acesso e rápida execução, que deve estar disponível em qualquer unidade de atendimento clínico. Através deste exame podem-se obter inúmeras informações úteis na abordagem destes pacientes intoxicados por ADT.

Por outro lado, outros instrumentos utilizados para avaliação clínica, como a dosagem dos níveis plasmáticos de ADT, são de custo elevado, execução demorada e restrita a laboratórios de grandes centros hospitalares.

Além destes motivos operacionais, quando comparados a sensibilidade e especificidade destes parâmetros eletrocardiográficos, principalmente a duração do QRS, com a dosagem sérica dos ADT, para avaliação do risco de complicações, principalmente de convulsões, não foram observadas grandes diferenças entre estes dois métodos, sendo que as respectivas áreas sobre a curva ROC ("Receiver-Operation Curve") foram similares. ${ }^{40,44,47}$

Os dados obtidos no presente estudo corroboram que os achados eletrocardiográficos são frequentes e reforçam esta tendência de utilizá-los no diagnóstico da intoxicação por ADT. ${ }^{41}$

O ECG também é um parâmetro utilizado para avaliação da indicação da utilização da alcalinização plasmática nos indivíduos intoxicados por ADT. A maior parte dos centros de intoxicação utiliza-se de um alargamento do complexo QRS maior que $100 \mathrm{~ms}$ como indicação para esta intervenção. ${ }^{43} \mathrm{~A}$ alcalinização plasmática, através da infusão endovenosa de bicarbonato de sódio, é o tratamento de escolha para estes pacientes com importantes alterações eletrocardiográficas; um dos mecanismos propostos é que a alcalinização plasmática desviaria o equilíbrio da droga para a sua forma ligada a albumina diminuindo a forma livre da mesma que é a responsável pela sua cardiotoxici- 
dade., ${ }^{2,42}$ Outro possível mecanismo seria o fornecimento de cargas elevadas de sódio através do bicarbonato de sódio diminuindo a intensidade do bloqueio dos canais rápido de sódio pela droga. ${ }^{2}$

Apesar da utilização do alargamento do complexo QRS maior que 100 ms como critério terapêutico, observou-se que sua prevalência foi baixa $(8,2 \mathrm{a}$ $21,3 \%$ ), embora com uma elevada probabilidade de complicações graves. Tais achados justificam as indicações, mas levanta a necessidade de se estudar melhor outros parâmetros eletrocardiográficos associados ao mesmo mecanismo fisiopatológico descrito que possam auxiliar na condução futura destes casos.

É importante reconhecer também que o ECG apresenta outras limitações nesta situação clínica. Além do alargamento do complexo QRS maior que 100 ms, outros parâmetros eletrocardiográficos apresentam uma acurácia moderada para predizer o risco de complicações como arritmias, convulsões e morte. ${ }^{40}$ Isto significa que alguns pacientes que apresentam estas alterações eletrocardiográficas, não apresentarão nenhuma complicação durante a evolução, já outros com ECG normal podem apresentar complicações clínicas graves. Alguns estudos recentes questionam a utilidade destes parâmetros eletrocardiográficos, principalmente a duração do complexo QRS, devido à baixa acurácia encontrada em análise retrospectiva de alguns centros de intoxicação. ${ }^{48,49,50,51}$
Esta limitação dos estudos pode ser decorrência do fato do ECG traduzir os efeitos da intoxicação de modo extremamente sensível e variável. A repetição do exame várias vezes durante a evolução do paciente, pode melhorar o desempenho deste teste diagnóstico e foi avaliada em poucos estudos. Um único estudo demonstrou que $80 \%$ dos pacientes tinham o máximo intervalo QRS registrado no ECG da admissão, mas os demais pacientes apresentaram o máximo intervalo QRS após 1 a 9 horas da admissão hospitalar, corroborando o fato de que as modificações são dinâmicas e novos estudos para documentar o impacto de monitorização eletrocardiográfica contínua são necessários. ${ }^{45,46}$

\section{Conclusão}

O ECG é um exame útil na avaliação dos pacientes com suspeita de intoxicação por ADT. O reconhecimento imediato das alterações eletrocardiográficas descritas acima acrescenta informações na seleção de um grupo de pacientes com maior risco de complicações e que necessitam de cuidados mais intensivos. Novos estudos prospectivos são necessários para melhor avaliação da acurácia destas variáveis eletrocardiográficas, como também, para melhor esclarecimento do impacto de exames seriados no desempenho deste exame complementar.

\section{ABSTRACT}

Introduction: The acute poisoning by tricyclic antidepressants is common in emergency rooms. Abnormalities in 12-lead electrocardiogram (ECG) are associated with this type of poisoning, with diagnostic and prognostic implications. The ECG may have incremental value especially in situations of altered consciousness, seizures or heart rhythm disturbances associated.

Objective: To review the electrocardiographic changes described in tricyclic antidepressant poisoning and to establish their prognostic implications in the management of this condition.

Methods: Bibliographic search in MEDLINE (PubMed) limited to articles published between 1980 and 2010 in English, using key words (tricyclic antidepressant overdose OR tricyclic antidepressant intoxication OR tricyclic antidepressant poisoning OR tricyclic antidepressant ingestion AND electrocardiography). Of the 133 articles identified, 44 were selected for review.

Results: We identified prevalent as changes with diagnostic power in descending order the following findings: deviation of $40 \mathrm{~ms}$ terminal of QRS to the right of the frontal plane (from 61.0 to $83.0 \%$ ), sinus tachycardia (40.7 to $57.7 \%$ ), QTc prolongation (8.2 to $53.0 \%$ ), ratio R / S in aVR> 0.7 (28.8 to $32.9 \%$ ), prolongation of QRS (8.2 to $21.3 \%$ ) and Brugada-like pattern (2.3 to $15.3 \%)$. Of these findings, only the QRS prolongation was prognostic for death, seizures and arrhythmias and QTc prolongation and the ratio $\mathrm{R}$ / $\mathrm{S}$ in $\mathrm{aVR}>0.7$ for ventricular arrhythmia. For the Brugada-like pattern, the evidence is sparse, but suggestive of a greater chance of seizures and hypotension.

Conclusion: The ECG is an instrument easily performed in the emergency room, providing diagnostic and prognostic information for poisoning by tricyclic and should be performed in all patients with suspicion of intoxication

Keywords: Antidepressants, Tricyclic. Overdose. Poisoning. Electrocardiography. 


\section{Referências}

1. McKenzie MS, McFarland BH. Trends in antidepressant overdoses. Pharmacoepidemiol Drug Saf 2007; 16:513-23.

2. Kerr GW, McGuffie AC, Wilkie S. Tricyclic antidepressant overdose: a review. Emerg Med J 2001; 18: 36-41.

3. Agrawal P, Nadel ES, Brown DFM. Tricyclic antidepressant overdose. J Emerg Med 2008; 34:321-5.

4. Langou RA, Van Dyke C, Tahan SR, Cohen LS. Cardiovascular manifestations of tricyclic antidepressant overdose. Am Heart J 1980; 100:458-64.

5. Marshall JB, Forker AD. Cardiovascular effects of tricyclic antidepressant drugs: therapeutic usage, overdose, and management of complications. Am Heart J 1982; 103:401-14.

6. Harrigan RA, Brady WJ. ECG Abnormalities in tricyclic antidepressant ingestion. Am J Emerg Med 1999; 17:387-93.

7. Fasoli RA, Glauser FL. Cardiac arrhythmias and ECG abnormalities in tricyclic antidepressant overdose. Clin Toxicol $1981 ; 18: 155-63$.

8. Cheng TO. ECG criteria for TCA toxicity. Ann Emerg Med $1990 ; 19: 219-20$

9. Veris-van Dieren J, Valk L, van Geijlswijk I, Tjan D, van Zanten A. Coma with ECG abnormalities: consider tricyclic antidepressant intoxication. Neth J Med 2007; 65:142-6.

10. Pellinen TJ, Färkkilä M, Heikkilä J, Luomanmäki K. Electrocardiographic an clinical features of tricyclic antidepressant intoxication. A survey of 88 cases and outlines of therapy. Ann Clin Res 1987; 19:12-7.

11. Niemann JT, Bessen HA, Rothstein RJ, Laks MM. Electrocardiographic criteria for tricyclic antidepressant cardiotoxicity. Am J Cardiol 1986, 57:1154-9.

12. Holstege CP, Eldridge DL, Rowden AK. ECG Manifestations: The Poisoned Patient. Emerg Med Clin North Am 2006; 24:15977.

13. Unverir P, Atilla R, Karciouglu O, Topacoglu H, Demiral Y,Tuncok $\mathrm{Y}$. A retrospective analysis of antidepressant poisoning in the emergency department: 11 year experience. Hum Exp Toxicol 2006; 25:605-12.

14. Mehta NJ, Alexandrou NA. Tricyclic antidepressant overdose an electrocardiographic changes. J Emerg Med 2000; 18: $463-4$.

15. Probst BD, Cetta F. The utility of a 12-lead electrocardiogram in diagnosing a suspected antidepressant overdose. Clin Pediatr 1992; 31:622-5.

16. Groleau G, Jotte R, Barish R. The electrocardiographic manifestations of cyclic antidepressant therapy and overdose: a review. J Emerg Med 1990; 8:597-605.

17. Wolfe TR, Caravati EM, Rollins DE.Terminal 40-ms frontal plane QRS Axis as a marker for tricyclic antidepressant overdose. Ann Emerg Med 1989; 18:348-51.

18. Berkovitch M, Matsui D, Fogelman R, Komar L, Hamilton R, Johnson D.Assessment of the terminal 40-millisecond QRS vector in children with a history of tricyclic antidepressant ingestion. Pediatr Emerg Care1995; 11:75-7.

19. Woolf $A D$, Francis PD, Woolf $A D$. ECG lead aVR versus $Q R S$ interval in predicting seizures and arrhythmias in acute tricyclic antidepressant toxicity. Ann Emerg Med 1995; 26:195-201.
20. Czartorysky B, Harding J. QRS interval in tricyclic antidepressant overdose. J Clin Psychiatry 1988; 49:456-7.

21. Boehnert MT, Lovejoy FH. Value of the QRS duration versus the serum drug level in predicting seizures and ventricular arrhythmias after an acute overdose of tricyclic antidepressants. N Engl J Med 1985; 313:474-9.

22. Shannon MW. Duration of QRS disturbances after severe tricyclic antidepressant intoxication. J Toxicol Clin Toxicol 1992; 30:377-86.

23. Olgun H, Yildirim ZK, Karacan M, Ceviz N. Clinical, electrocardiographic, and laboratory findings in children with amitriptyline intoxication. Pediatr Emerg Care 2009; 25: 170-3.

24. Al-Khatib SM, LaPointe NMA, Kramer JM, Califf RM. What clinicians should know about the QT interval. JAMA 2003; 289:2120-7

25. Belardinelli L, Antzelevitch C, Vos MA. Assessing predictors of drug-induced torsade de pointes. Trends Pharmacol Sci 2003; 24:619-25.

26. Vieweg WV, Wood MA. Tricyclic antidepressants, QT interval prolongation, and torsade de pointes. Psychosomatics 2004; 45:371-7.

27. Davison ET. Amitriptyline-induced Torsade de Pointes. Successful therapy with atrial pacing. J Electrocardiol 1985; 18:299-301.

28. Sala M, Coppa F, Cappucciati C, Brambilla P, D’Allio G, Caverzasi E, Barale F, De Ferrrani GM. Antidepressants: their effects on cardiac channels, QT prolongation and torsades de pointes. Curr Opin Investig Drugs 2006; 7:256-63.

29. Herrmann HC, Kaplan LM, Bierer BE. Q-T prolongation and torsades de pointes ventricular tachycardia produced by the tetracyclic antidepressant agent maprotiline. Am J Cardiol 1983; $51: 904-6$

30. Antzelevitch C, Brugada P, Brugada J, Brugada R. Brugada syndrome: from cell to bedside. Curr Probl Cardiol 2005; 30:9-54

31. Tada H, Sticherling C, Oral H, Morady F. Brugada syndrome mimicked by tricyclic antidepressant overdose. J Cardiovasc Electrophysiol 2001; 12:275.

32. Goldgran-Toledano D, Sideris G, Kevorkian J-P. Overdose of cyclic antidepressants and the Brugada syndrome. N Engl J Med 2002; 346:1591-2.

33. Bigwood B, Galler D, Amir N, Smith W. Brugada syndrome following tricyclic antidepressant overdose. Anaesth Intensive Care 2005; 33:266-70

34. Rouleau F, Asfar P, Boulet S, et al. Transient ST segment elevation in right precordial leads induced by psychotropic drugs: relationship to the Brugada syndrome. J Cardiovas Electrophysiol 2001; 12:61-5.

35. Akhtar M, Goldschlager NF. Brugada electrocardiographic pattern due to tryciclic antidepressant overdose. J Electrocardiol 2006; 39:336-9.

36. Monteban-Kooistra WE, van den Berg MP, Tulleken JE, Ligtenberg JJ, Meertens JH, Zijlstra JG. Brugada electrocardiographic pattern elicited by cyclic antidepressants overdose. Intensive Care Med 2006; 32:281-5.

37. Berbata VS, Phillips S, Eberhardt A, Calihan KJ, Waksman JC, Hear K. Incidence of Brugada electrocardiographic pattern and outcomes of these patients after intentional tricyclic antidepressant ingestion. Am J Cardiol 2007; 100:656-60. 
38. Peters RW, Buser GA, Kim HJ, Gold MR. Tricyclic overdose causing sustained monomorphic ventricular tachycardia. Am J Cardiol 1992; 70:1226-8.

39. Pang PS, Look RB, Brown DF, Nadel ES. Wide complex rhythm and cardiac arrest. J Emerg Med 2004; 26:197-200.

40. Bailey B, Buckley NA, Amre DK. A meta-analysis of prognostic indicators to predict seizures, arrhythmias or death after tricyclic antidepressant overdose. J Toxicol Clin Toxicol 2004; 42:877-88.

41. Caravati EM. The electrocardiogram as a diagnostic discriminator for acute tricyclic antidepressant poisoning. J Toxicol Clin Toxicol 1999; 37:113-5.

42. Chan CYY, Waring S. Tricyclic cardiotoxicity treated with sodium bicarbonate. Circulation 2007; 115:e63-4.

43. Seger DL, Hantsch C, Zavoral T, Wrenn K. Variability of recommendations for serum alkalinization in tricyclic antidepressant overdose: a survey of U.S. poison center medical directors. J Toxicol Clin Toxicol 2003; 41:331-8.

44. Johnson DA, Knepp IG, Whelan TV. Toxic tricyclic antidepressant levels and the ECG. JAMA 1983; 250:1027.

45. Liebelt EL, Ulrich A, Francis PD, Woolf A. Serial electrocardiogram changes in acute tryciclic antidepressant overdoses. Crit Care Med 1997; 25:1721-6.
46. Singh N, Singh HK, Khan IA. Serial electrocardiographic changes as a predictor of cardiovascular toxicity in acute tricyclic antidepressant overdose. Am J Ther 2002; 9:75-9.

47. Lavoie FW, Gansert GG, Weiss RE. Value of inicial ECG findings and plasma drug levels in cyclic antidepressant overdose. Ann Emerg Med 1990; 19:696-700.

48. Buckley NA, Chevalier S,Leditschke A, O'Connell DL, Leitch J, Ponds S. The limited utility of electrocardiography variables used to predict arrhythmias in psychotropic drug overdose. Crit Care 2003; 7:R101-7.

49. Buckley NA, O'Connell DL, Whyte IM, Dawson AH. Interrater agreement in the measurement of QRS interval in tricyclic antidepressant overdose: implications for monitoring and research. Ann Emerg Med 1996; 28:515-9.

50. Emerson TS. Inaccuracy of QRS interval as TCA toxicity indicator. Ann Emerg Med 1987; 16:1312-3.

51. Foulke GE, Albertson TE. QRS interval in tricyclic antidepressant overdosage: inaccuracy as a toxicity indicator in emergency settings. Ann Emerg Med 1987; 16:160-3. 